\title{
ON THE EPSTEIN ZETA FUNCTION
}

\section{ZHANG NAN-YUE AND KENNETH S. WILLIAMS}

Abstract. The Epstein zeta function $Z(s)$ is defined for Re $s>1$ by

$$
Z(s)=\sum_{\substack{m, n=-\infty \\(m, n) \neq(0,0)}}^{\infty} \frac{1}{\left(a m^{2}+b m r+c n^{2}\right)^{s}}
$$

where $a, b, c$ are real numbers with $a>0$ and $b^{2}-4 a c<0 . Z(s)$ can be continued analytically to the whole complex plane except for a simple pole at $s=1$. Simple proofs of the functional equation and of the Kronecker "Grenz-formel" for $Z(s)$ are given. The value of $Z(k)(k=2,3, \ldots)$ is determined in terms of infinite series of the form

$$
\sum_{n=1}^{\infty} \frac{\cot ^{r} n \pi \tau}{n^{2 k-1}}(r=1,2, \ldots, k)
$$

where $\tau=\left(b+\sqrt{b^{2}-4 a c}\right) / 2 a$.

\section{Introduction}

Let $a, b$ and $c$ be real numbers with $a>0$ and $D=4 a c-b^{2}>0$, so that

$$
Q(u, v)=a u^{2}+b u v+c v^{2}
$$

is a positive-definite binary quadratic form of discriminant $-D$.

The Epstein zeta function $Z(s)$ is defined by the double series

$$
Z(s)=\sum_{\substack{m, n=-\infty \\(m, n) \neq(0,0)}}^{\infty} \frac{1}{Q(m, n)^{s}},
$$

Received January 18, 1994.

1991 Mathematics Subject Classification. 11M41.

Key words and phrases. Epstein zeta function, functional equation, Kronecker Grenz-formel.

(1) Research supported by the National Natural Science Foundation of China.

(2) Research supported by Natural Sciences and Engineering Research Council of Canada Grant A-7233. 
where $s=\sigma+i t$ and $\sigma, t$ are real numbers with $\sigma>1$.

Since $Q(u, v) \geq \lambda\left(u^{2}+v^{2}\right)$ with

$$
\lambda=\frac{1}{2}\left(a+c-\sqrt{(a-c)^{2}+b^{2}}\right)>0,
$$

for all real numbers $u$ and $v$, the series (1.2) converges absolutely for $\sigma>1$ and uniformly in every half plane $\sigma \geq 1+\epsilon(\epsilon>0)$. Thus $Z(s)$ is an analytic function of $s$ for $\sigma>1$. Furthermore, the function $Z(s)$ can be continued analytically to the whole complex plane except for a simple pole at $s=1$ and satisfies the functional equation

$$
\left(\frac{\sqrt{D}}{2 \pi}\right)^{s} \Gamma(s) Z(s)=\left(\frac{\sqrt{D}}{2 \pi}\right)^{1-s} \Gamma(1-s) Z(1-s) .
$$

The purpose of this paper is three-fold. First, in $\S 2$, by making use of the Poisson summation formula and an integral representation of the Bessel function, we give a proof of the functional equation (1.3), which in the authors' view is simpler than those given in [1], [4], [7]. Secondly, in $\S 3$, we deduce, in a very simple manner from the results of $\S 2$, the values of $A_{-1}$ and $A_{0}$ in the Laurent expansion

$$
Z(s)=\frac{A_{-1}}{s-1}+A_{0}+A_{1}(s-1)+\cdots,
$$

valid in a neighbourhood of $s=1$ (the so-called Kronecker "Grenz-formel"). Again we believe our proof to be more direct than those in [3], [4] and [5]. Finally, in $\S 4$, we determine the value of $Z(k)$ for any positive integer $k \geq 2$ in terms of series of the type $\sum_{n=1}^{\infty} \frac{\cot ^{r} n \pi \tau}{n^{2 k-1}}$, where $\tau=(b+i \sqrt{D}) / 2 a$ and $r=1, \ldots, k$. The reader should compare our result with that of Smart [6].

\section{The Functionall Equation of $Z(s)$.}

Setting

$$
x=\frac{b}{2 a}, \quad y=\frac{\sqrt{D}}{2 a}, \quad \tau=x+i y=\frac{b+i \sqrt{D}}{2 a}
$$

we have

$$
\tau+\bar{\tau}=2 x=\frac{b}{a}, \quad \tau \bar{\tau}=\frac{b^{2}+D}{4 a^{2}}=\frac{c}{a}
$$

so that

$$
Q(m, n)=a m^{2}+b m n+c n^{2}=a(m+n \tau)(m+n \bar{\tau})=a|m+n \tau|^{2}
$$

and

$$
Z(s)=\sum_{\substack{m, n=-\infty \\(m, n) \neq(0,0)}}^{\infty} \frac{1}{a^{s}|m+n \tau|^{2 s}}, \quad \sigma>1
$$


Separating the term with $n=0$, we obtain

$$
Z(s)=\frac{2}{a^{s}} \sum_{m=1}^{\infty} \frac{1}{m^{2 s}}+\frac{2}{a^{s}} \sum_{n=1}^{\infty} \sum_{m=-\infty}^{\infty} \frac{1}{|m+n \tau|^{2 s}}, \quad \sigma>1 .
$$

In order to evaluate the second term in (2.3), we apply the Poisson summation formula $[8, \mathrm{p} .17]$

$$
\sum_{m=-\infty}^{\infty} f(m)=\sum_{m=-\infty}^{\infty} \int_{-\infty}^{\infty} f(u) \cos 2 m \pi u d u
$$

to the function $f(t)=\frac{1}{|t+\tau|^{2 s}}$ and obtain

$$
\begin{aligned}
\sum_{m=-\infty}^{\infty} \frac{1}{|m+\tau|^{2 s}} & =\sum_{m=-\infty}^{\infty} \int_{-\infty}^{\infty} \frac{\cos 2 m \pi u}{|u+\tau|^{2 s}} d u \\
& =\sum_{m=-\infty}^{\infty} \int_{-\infty}^{\infty} \frac{\cos 2 m \pi u}{\left\{(u+x)^{2}+y^{2}\right\}^{s}} d u=\sum_{m=-\infty}^{\infty} \int_{-\infty}^{\infty} \frac{\cos 2 m \pi(t-x)}{\left(t^{2}+y^{2}\right)^{s}} d t \\
& =\sum_{m=-\infty}^{\infty} \cos 2 m \pi x \int_{-\infty}^{\infty} \frac{\cos 2 m \pi t}{\left(t^{2}+y^{2}\right)^{s}} d t
\end{aligned}
$$

since the integrals involving the sine function vanish, that is

$$
\begin{aligned}
& \sum_{m=-\infty}^{\infty} \frac{1}{|m+\tau|^{2 s}} \\
= & \frac{2}{y^{2 s-1}} \int_{0}^{\infty} \frac{1}{\left(1+t^{2}\right)^{s}} d t+\frac{2^{2}}{y^{2 s-1}} \sum_{m=1}^{\infty} \cos 2 m \pi x \int_{0}^{\infty} \frac{\cos 2 m \pi y t}{\left(1+t^{2}\right)^{s}} d t, \quad \sigma>1 .
\end{aligned}
$$

Next, we evaluate the two integrals appearing in (2.5). Making the substitution $u=\frac{t^{2}}{1+t^{2}}$, we have

$$
\frac{1}{1+t^{2}}=1-u, \quad d u=\frac{2 t d t}{\left(1+t^{2}\right)^{2}}=2 u^{1 / 2}(1-u)^{3 / 2} d t
$$

and

$$
\begin{aligned}
\int_{0}^{\infty} \frac{d t}{\left(1+t^{2}\right)^{s}} & =\frac{1}{2} \int_{0}^{1}(1-u)^{s-3 / 2} u^{-1 / 2} d u=\frac{1}{2} B\left(s-\frac{1}{2}, \frac{1}{2}\right) \\
& =\frac{\Gamma\left(s-\frac{1}{2}\right) \Gamma\left(\frac{1}{2}\right)}{2 \Gamma(s)}=\frac{\Gamma\left(s-\frac{1}{2}\right) \sqrt{\pi}}{2 \Gamma(s)} .
\end{aligned}
$$

From an integral representation of the Bessel function [2, p.140]

$$
K_{\nu}(y)=\frac{1}{\sqrt{\pi}}\left(\frac{2}{y}\right)^{\nu} \Gamma\left(\nu+\frac{1}{2}\right) \int_{0}^{\infty} \frac{\cos y t}{\left(1+t^{2}\right)^{\nu+\frac{1}{2}}} d t, \quad y>0, \quad \operatorname{Re} \nu>-\frac{1}{2}
$$


we have

$$
\int_{0}^{\infty} \frac{\cos 2 m \pi y t d t}{\left(1+t^{2}\right)^{s}}=\frac{\sqrt{\pi}(m \pi y)^{s-\frac{1}{2}}}{\Gamma(s)} K_{s-\frac{1}{2}}(2 m \pi y), \quad \sigma>\frac{1}{2} .
$$

Putting (2.6) and (2.8) into (2.5), we obtain

$$
\begin{aligned}
& \sum_{m=-\infty}^{\infty} \frac{1}{\sqrt{m+\left.\tau\right|^{2 s}}} \\
= & \frac{\Gamma\left(s-\frac{1}{2}\right) \sqrt{\pi}}{y^{2 s-1} \Gamma(s)}+\frac{4 \sqrt{m}}{y^{2 s-1} \Gamma(s)} \sum_{m=1}^{\infty}(m \pi y)^{s-\frac{1}{2}} \cos (2 m \pi x) K_{s-\frac{1}{2}}(2 m \pi y), \quad \sigma>1,
\end{aligned}
$$

so that for $n \geq 1$

$$
\begin{aligned}
& \sum_{m=-\infty}^{\infty} \frac{1}{|m+n \tau|^{2 s}} \\
= & \frac{\Gamma\left(s-\frac{1}{2}\right) \sqrt{\pi}}{n^{2 s-1} y^{2 s-1} \Gamma(s)}+\frac{4 \sqrt{\pi}}{n^{2 s-1} y^{2 s-1} \Gamma(s)} \sum_{m=1}^{\infty}(m n \pi y)^{s-\frac{1}{2}} \cos (2 m n \pi x) K_{s-\frac{1}{2}}(2 m n \pi y) .
\end{aligned}
$$

Then, from (2.9) and (2.3), we have

$$
\begin{aligned}
Z(s)= & 2 a^{-s} \zeta(2 s)+2 a^{-s} y^{1-2 s} \frac{\Gamma\left(s-\frac{1}{2}\right) \sqrt{\pi}}{\Gamma(s)} \zeta(2 s-1) \\
& +\frac{8 a^{-s} y^{\frac{1}{2}-s} \pi^{s}}{\Gamma(s)} \sum_{n=1}^{\infty} n^{1-2 s} \sum_{m=1}^{\infty}(m n)^{s-\frac{1}{2}} \cos (2 m n \pi x) K_{s-\frac{1}{2}}(2 m n \pi y) .
\end{aligned}
$$

Collecting the terms with $m n=k$, we obtain

$$
\begin{aligned}
Z(s)= & 2 a^{-s} \zeta(2 s)+2 a^{-s} y^{1-2 s} \frac{\Gamma\left(s-\frac{1}{2}\right) \sqrt{\pi}}{\Gamma(s)} \zeta(2 s-1) \\
& +\frac{8 a^{-s} y^{\frac{1}{2}-s} \pi^{s}}{\Gamma(s)} \sum_{k=1}^{\infty}\left(\sum_{n \mid k} n^{1-2 s}\right) k^{s-\frac{1}{2}} \cos (2 k \pi x) K_{s-\frac{1}{2}}(2 k \pi y),
\end{aligned}
$$

that is

$$
Z(s)=2 a^{-s} \zeta(2 s)+2 a^{-s} y^{1-2 s} \frac{\Gamma\left(s-\frac{1}{2}\right) \sqrt{\pi}}{\Gamma(s)} \zeta(2 s-1)+\frac{2 a^{-s} y^{\frac{1}{2}-s} \pi^{s}}{\Gamma(s)} H(s),
$$

where

$$
H(s)=4 \sum_{k=1}^{\infty} \sigma_{1-2 s}(k) k^{s-\frac{1}{2}} \cos (2 k \pi x) K_{s-\frac{1}{2}}(2 k \pi y)
$$


and $\sigma_{\nu}(k)$ denotes the sum of the $\nu$-th powers of the divisors of $k$, that is,

$$
\sigma_{\nu}(k)=\sum_{d \mid k} d^{\nu}=\sum_{d \mid k}\left(\frac{k}{d}\right)^{\nu}
$$

The formula (2.11) provides the analytic continuation of $Z(s)$. In fact, the first two terms on the right-side of (2.11) have removable singularities at $s=\frac{1}{2}-n(n=0,1,2, \ldots)$ because at $s=\frac{1}{2}$ the pole of $\zeta(2 s)$ is cancelled by the pole of $\Gamma\left(s-\frac{1}{2}\right)$ and at $s=\frac{1}{2}-n$ $(n=1,2, \ldots)$ the pole of $\Gamma\left(s-\frac{1}{2}\right)$ is cancelled by the zero of $\zeta(2 s-1)(\zeta(-2 n)=0$, $n=1,2, \ldots)$; besides, it is not difficult to prove that $H(s)$ is an entire function [1]. Thus, it follows the $Z(s)$ has a continuation in the whole finite complex plane except for a simple pole at $s=1$.

Now, we write (2.11) in another form:

$$
\left(\frac{a y}{\pi}\right)^{s} \Gamma(s) Z(s)=2\left(\frac{y}{\pi}\right)^{s} \Gamma(s) \zeta(2 s)+2 y^{1-s} \pi^{\frac{1}{2}-s} \Gamma\left(s-\frac{1}{2}\right) \zeta(2 s-1)+2 y^{\frac{1}{2}} H(s) .
$$

By the functional equation for the Riemann zeta function

$$
\zeta(2 s-1)=2(2 \pi)^{2 s-2} \sin \left(s-\frac{1}{2}\right) \pi \Gamma(2-2 s) \zeta(2-2 s)
$$

and the basic properties of the gamma function

$$
\begin{aligned}
\Gamma(2-2 s) & =\frac{\Gamma(1-s) \Gamma\left(\frac{3}{2}-s\right)}{\sqrt{\pi} 2^{2 s-1}}=\frac{\Gamma(1-s)}{\sqrt{\pi} 2^{2 s-1}} \cdot \frac{\pi}{\Gamma\left(s-\frac{1}{2}\right) \sin \left(s-\frac{1}{2}\right) \pi} \\
& =\frac{\sqrt{\pi} \Gamma(1-s)}{2^{2 s-1} \Gamma\left(s-\frac{1}{2}\right) \sin \left(s-\frac{1}{2}\right) \pi}
\end{aligned}
$$

we have

$$
\pi^{\frac{1}{2}-s} \Gamma\left(s-\frac{1}{2}\right) \zeta(2 s-1)=\pi^{s-1} \Gamma(1-s) \zeta(2-2 s)
$$

and

$$
\left(\frac{a y}{\pi}\right)^{s} \Gamma(s) Z(s)=2\left(\frac{y}{\pi}\right)^{s} \Gamma(s) \zeta(2 s)+2\left(\frac{y}{\pi}\right)^{1-s} \Gamma(1-s) \zeta(2-2 s)+2 y^{\frac{1}{2}} H(s) .
$$

Recalling the following facts:

$$
K_{-\nu}(y)=K_{\nu}(y)
$$

(see $[2, \mathrm{p} .110]$ ) and

$$
k^{-\frac{\nu}{2}} \sigma_{\nu}(k)=k^{-\frac{\nu}{2}} \sum_{d \mid k} d^{\nu}=k^{-\frac{\nu}{2}} \sum_{d \mid k}\left(\frac{k}{d}\right)^{\nu}=k^{\frac{\nu}{2}} \sigma_{-\nu}(k)
$$


we have from (2.12)

$$
H(s)=H(1-s)
$$

If we put

$$
\phi(s)=\left(\frac{a y}{\pi}\right)^{s} \Gamma(s) Z(s)
$$

then from (2.14) and (2.15) we obtain

$$
\phi(s)=\phi(1-s)
$$

Since $a y=\sqrt{D} / 2$ (from $(2.1)),(2.17)$ can be rewritten in the following form

$$
\left(\frac{\sqrt{D}}{2 \pi}\right)^{s} \Gamma(s) Z(s)=\left(\frac{\sqrt{D}}{2 \pi}\right)^{1-s} \Gamma(1-s) Z(1-s)
$$

which is the functional equation of $Z(s)$.

\section{Determination of $A_{-1}$ and $A_{0}$.}

As $Z(s)$ is an analytic function in the finite complex plane except for a simple pole at $s=1, Z(s)$ has a Laurent expansion

$$
Z(s)=\frac{A_{-1}}{s-1}+A_{0}+A_{1}(s-1)+\cdots
$$

valid in a neighbourhood of $s=1$. In this neighbourhood of $s=1$, we have the following expansions:

$$
\zeta(2 s-1)=\frac{1}{2(s-1)}+\gamma+\cdots,
$$

where $\gamma$ denotes Euler's constant,

$$
\begin{aligned}
\frac{\Gamma\left(s-\frac{1}{2}\right) \sqrt{\pi}}{\Gamma(s)} & =\pi+\left.\pi^{\frac{1}{2}}\left[\frac{\Gamma\left(s-\frac{1}{2}\right)}{\Gamma(s)}\right]^{\prime}\right|_{s=1}(s-1)+\cdots \\
& =\pi+\pi^{\frac{1}{2}}\left(\psi\left(\frac{1}{2}\right)+\gamma\right) \Gamma\left(\frac{1}{2}\right)(s-1)+\cdots \quad\left(\text { where } \psi(s)=\frac{\Gamma^{\prime}(s)}{\Gamma(s)}\right) \\
& =\pi-2 \pi \log 2 \cdot(s-1)+\cdots,
\end{aligned}
$$

since $\psi(1)=-\gamma$ and $\psi\left(\frac{1}{2}\right)=-\gamma-2 \log 2$;

$$
2 a^{-s} y^{1-s}=\frac{2}{a y}\left[1-\log \left(a y^{2}\right)(s-1)+\cdots\right]
$$


and

$$
\begin{aligned}
& 2 a^{-s} y^{1-2 s} \frac{\Gamma\left(s-\frac{1}{2}\right) \sqrt{\pi}}{\Gamma(s)} \zeta(2 s-1) \\
= & \frac{2 \pi}{a y}\left[1-\log \left(a y^{2}\right)(s-1)+\cdots\right][1-2 \log 2(s-1)+\cdots]\left[\frac{1}{2(s-1)}+\gamma+\cdots\right] \\
= & \frac{4 \pi}{\sqrt{D}}\left[1-\log \left(a y^{2}\right)(s-1)+\cdots\right]\left[\frac{1}{2(s-1)}+(\gamma-\log 2)+\cdots\right] \\
= & \frac{4 \pi}{\sqrt{D}}\left[\frac{1}{2(s-1)}+\left(\gamma-\log 2-\frac{1}{2} \log \left(a y^{2}\right)\right)+\cdots\right] \\
= & \frac{2 \pi}{\sqrt{D}} \cdot \frac{1}{s-1}+\frac{2 \pi}{\sqrt{D}}\left(2 \gamma-\log \frac{D}{a}\right)+\cdots .
\end{aligned}
$$

From (3.2) and (2.11), we obtain

$$
Z(s)=\frac{\pi^{2}}{3 a}+\frac{2 \pi}{\sqrt{D}} \cdot \frac{1}{s-1}+\frac{2 \pi}{\sqrt{D}}\left(2 \gamma-\log \frac{D}{a}\right)+2 a^{-1} y^{-\frac{1}{2}} \pi H(1)+O(|s-1|) .
$$

Next, we evaluate the fourth term on the right side of (3.3) which contains $H(1)$. We have

$$
\begin{aligned}
2 a^{-1} y^{-\frac{1}{2}} \pi H(1) & =8 a^{-1} y^{-\frac{1}{2}} \pi \sum_{k=1}^{\infty} \sigma_{-1}(k) k^{\frac{1}{2}} \cos (2 \pi k x) K_{\frac{1}{2}}(2 \pi k y) \\
& =8 a^{-1} y^{-1} \sum_{k=1}^{\infty} \sigma_{-1}(k) \cos (2 \pi k x) \int_{0}^{\infty} \frac{\cos 2 \pi k y t}{1+t^{2}} d t \quad(\text { by }(2.7)) \\
& =\frac{8 \pi}{\sqrt{D}} \sum_{k=1}^{\infty} \sigma_{-1}(k) \cos (2 \pi k x) e^{-2 \pi k y} \quad\left(\text { as } \int_{0}^{\infty} \frac{\cos l t}{1+t^{2}} d t=\frac{\pi}{2} e^{-l}\right) .
\end{aligned}
$$

If we set $q=e^{\pi i \tau}$ (so that $|q|=e^{-\pi y}<1$ as $y>0$ ) then $q^{2 k}+\bar{q}^{2 k}=2 e^{-2 k \pi y} \cos 2 k \pi x$ and.

$$
\begin{aligned}
2 a^{-1} y^{-\frac{1}{2}} \pi H(1)= & \frac{4 \pi}{\sqrt{D}}\left(\sum_{k=1}^{\infty} \sigma_{-1}(k) q^{2 k}+\sum_{k=1}^{\infty} \sigma_{-1}(k) \bar{q}^{2 k}\right) \\
& =-\frac{4 \pi}{\sqrt{D}} \log \prod_{k=1}^{\infty}\left(1-q^{2 k}\right)\left(1-\bar{q}^{2 k}\right) \\
& =-\frac{4 \pi}{\sqrt{D}} \log \prod_{k=1}^{\infty}\left|1-q^{2 k}\right|^{2},
\end{aligned}
$$

and from (3.3) we have

$$
Z(s)=\frac{\pi^{2}}{3 a}+\frac{2 \pi}{\sqrt{D}} \cdot \frac{1}{s-1}+\frac{2 \pi}{\sqrt{D}}\left(2 \gamma-\log \frac{D}{a}\right)-\frac{4 \pi}{\sqrt{D}} \log \prod_{k=1}^{\infty}\left|1-q^{2 k}\right|^{2}+O(|s-1|)
$$


The Dedekind eta function $\eta(z)$ is given by

$$
\eta(z)=e^{\frac{\pi i z}{12}} \prod_{n=1}^{\infty}\left(1-e^{2 \pi i n z}\right), \quad z=x+i y, \quad y>0 .
$$

Hence we have

$$
\log \prod_{n=1}^{\infty}\left|1-q^{2 n}\right|^{2}=\log |\eta(\tau)|^{2}+\frac{\pi y}{6}=2 \log |\eta(\tau)|+\frac{\pi \sqrt{D}}{12 a}
$$

giving another form of (3.4):

$$
Z(s)=\frac{2 \pi}{\sqrt{D}} \cdot \frac{1}{s-1}+\frac{2 \pi}{\sqrt{D}}\left(2 \gamma-\log \frac{D}{a}\right)-\frac{8 \pi}{\sqrt{D}} \log |\eta(\tau)|+O(|s-1|) .
$$

We have shown that

$$
A_{-1}=\frac{2 \pi}{\sqrt{D}}, \quad A_{0}=\frac{4 \pi \gamma}{\sqrt{D}}-\frac{2 \pi}{\sqrt{D}} \log \frac{D}{a}-\frac{8 \pi}{\sqrt{D}} \log \left|\eta\left(\frac{b+i \sqrt{D}}{2 a}\right)\right| .
$$

\section{Evaluation of $Z(k)$ for any positive integer $k \geq 2$.}

We need the following lemma. $\lambda$,

Lemma. For any non-negative integer $k$ and any non-negative real number

$$
I_{k}(\lambda)=\int_{0}^{\infty} \frac{\cos 2 \pi \lambda x}{\left(1+x^{2}\right)^{k+1}} d x=\frac{\pi}{2^{2 k+1}} e^{-2 \pi \lambda} \sum_{j=0}^{k}\left(\begin{array}{c}
2 k-j \\
k
\end{array}\right)(4 \pi \lambda)^{j} / j !
$$

Proof. Let $C_{R}$ denote the contour in the upper half of the complex $z$-plane consisting of the semi-circle $|z|=R$ and the real axis from $z=-R$ to $z=R$. Applying Cauchy's residue theorem to the integral of the function $\frac{e^{2 \pi i \lambda z}}{\left(1+z^{2}\right)^{k+1}}$ along the contour $C_{R}$, and then letting $R \rightarrow+\infty$, we obtain

$$
\begin{aligned}
I_{k}(\lambda) & =\frac{1}{2} \int_{-\infty}^{\infty} \frac{e^{2 \pi i \lambda x}}{\left(1+x^{2}\right)^{k+1}} d x=\frac{1}{2} \cdot 2 \pi i \cdot \frac{1}{k !} \frac{d^{k+1}}{d z^{k+1}}\left\{\frac{e^{2 \pi i \lambda z}}{(z+i)^{k+1}}\right\}_{z=i} \\
& =\frac{\pi i}{k !} \sum_{j=0}^{k}\left(\begin{array}{c}
k \\
j
\end{array}\right)\left(e^{2 \pi i \lambda z}\right)_{z=i}^{(j)}\left(\frac{1}{(z+i)^{k+1}}\right)_{z=i} \\
& =\frac{\pi i}{k !} \sum_{j=0}^{k}\left(\begin{array}{c}
k \\
j
\end{array}\right)(2 \pi i \lambda)^{j} e^{-2 \pi \lambda} \frac{(k+1)(k+2) \cdots(k+k-j)}{(2 i)^{2 k-j+1}}(-1)^{k-j} \\
& =\frac{\pi}{2^{2 k+1}} e^{-2 \pi \lambda} \sum_{j=0}^{k}\left(\begin{array}{c}
2 k-j \\
k
\end{array}\right)(4 \pi \lambda)^{j} / j !
\end{aligned}
$$


Now we start the evaluation of $Z(k+1), k \geq 1$. First, from (2.5), we have

$$
\sum_{m=-\infty}^{\infty} \frac{1}{|m+\tau|^{2 k+2}}=\frac{2}{y^{2 k+1}} \int_{0}^{\infty} \frac{d t}{\left(1+t^{2}\right)^{k+1}}+\frac{4}{y^{2 k+1}} \sum_{m=1}^{\infty} \cos (2 m \pi x) I_{k}(m y) .
$$

Then, by (2.6) and the lemma, we deduce that

$$
\begin{aligned}
& \sum_{m=-\infty}^{\infty} \frac{1}{|m+\tau|^{2 k+2}} \\
= & \frac{\Gamma\left(k+\frac{1}{2}\right) \sqrt{\pi}}{y^{2 k+1} k !}+\frac{4 \pi}{(2 y)^{2 k+1}} \sum_{j=0}^{k}\left(\begin{array}{c}
2 k-j \\
k
\end{array}\right) \frac{1}{j !} \sum_{m=1}^{\infty}(4 \pi m y)^{j} \cos (2 m \pi x) e^{-2 m \pi y} \\
= & \frac{2 \pi}{(2 y)^{2 k+1}}\left(\begin{array}{c}
2 k \\
k
\end{array}\right)+\frac{4 \pi}{(2 y)^{2 k+1}} \sum_{j=0}^{k}\left(\begin{array}{c}
2 k-j \\
k
\end{array}\right) \frac{1}{j !} \sum_{m=1}^{\infty}(4 \pi m y)^{j} \cos (2 m \pi x) e^{-2 m \pi y} .
\end{aligned}
$$

Putting the above equality into (2.3), we obtain

$$
\begin{aligned}
& \frac{1}{2} a^{k+1} Z(k+1) \\
= & \zeta(2 k+2)+\frac{2 \pi}{(2 y)^{2 k+1}}\left(\begin{array}{c}
2 k \\
k
\end{array}\right) \zeta(2 k+1) \\
& +\frac{4 \pi}{(2 y)^{2 k+1}} \sum_{j=0}^{k}\left(\begin{array}{c}
2 k-j \\
k
\end{array}\right) \frac{1}{j !}(4 \pi y)^{j} \sum_{n=1}^{\infty} \frac{1}{n^{2 k+1}} \sum_{m=1}^{\infty}(m n)^{j} \cos (2 m n \pi x) e^{-2 m n \pi y} .
\end{aligned}
$$

Since

$$
\begin{aligned}
\cos (2 m n \pi x) e^{-2 m n \pi y} & =\frac{1}{2}\left(e^{2 m n \pi i \tau}+e^{-2 m n \pi i \bar{\tau}}\right) \\
& =\frac{1}{2}(e(m n \tau)+e(-m n \bar{\tau})),
\end{aligned}
$$

we have

$$
\begin{aligned}
& \frac{1}{2} a^{k+1} Z(k+1) \\
= & \zeta(2 k+2)+\frac{2 \pi}{(2 y)^{2 k+1}}\left(\begin{array}{c}
2 k \\
k
\end{array}\right) \zeta(2 k+1) \\
+ & \frac{2 \pi}{(2 y)^{2 k+1}} \sum_{j=0}^{k}\left(\begin{array}{c}
2 k-j \\
k
\end{array}\right) \frac{1}{j !}(4 \pi y)^{j} \sum_{n=1}^{\infty} \frac{1}{n^{2 k+1}} \sum_{m=1}^{\infty}(m n)^{j}(e(m n \tau)+e(-m n \bar{\tau})) .
\end{aligned}
$$

In view of the fact that if $\operatorname{Im} \tau>0$,

$$
\left\{\begin{array}{l}
\sum_{m=1}^{\infty} e(m \tau)=\frac{i}{2} \cot \pi \tau-\frac{1}{2}, \\
\sum_{m=1}^{\infty} e(-m \bar{\tau})=\frac{-i}{2} \cot \pi \bar{\tau}-\frac{1}{2},
\end{array}\right.
$$


we have

$$
\sum_{m=1}^{\infty}(e(m \tau)+(-m \bar{\tau}))=\frac{i}{2}(\cot \pi \tau-\cot \pi \bar{\tau})-1
$$

From (4.2) and (4.4), we have

$$
\begin{aligned}
& \frac{1}{2} a^{k+1} Z(k+1) \\
= & \zeta(2 k+2)+\frac{2 \pi}{(2 y)^{2 k+1}}\left(\begin{array}{c}
2 k \\
k
\end{array}\right)\left[\zeta(2 k+1)+\sum_{n=1}^{\infty} \frac{1}{n^{2 k+1}} \sum_{m=1}^{\infty}((e(m n \tau)+e(-m n \bar{\tau}))]\right. \\
& +\frac{2 \pi}{(2 y)^{2 k+1}} \sum_{j=1}^{k}\left(\begin{array}{c}
2 k-j \\
k
\end{array}\right) \frac{1}{j !}(4 \pi y)^{j} \sum_{n=1}^{\infty} \frac{1}{n^{2 k+1}} \sum_{m=1}^{\infty}(m n)^{j}(e(m n \tau)+e(-m n \bar{\tau})) \\
= & \zeta(2 k+2)+\frac{\pi i}{(2 y)^{2 k+1}}\left(\begin{array}{c}
2 k \\
k
\end{array}\right) \sum_{n=1}^{\infty} \frac{1}{n^{2 k+1}}(\cot \pi n \tau-\cot \pi n \bar{\tau}) \\
& +\frac{2 \pi}{(2 y)^{2 k+1}} \sum_{j=1}^{k}\left(\begin{array}{c}
2 k-j \\
k
\end{array}\right) \frac{1}{j !}(4 \pi y)^{j} \sum_{n=1}^{\infty} \frac{1}{n^{2 k+1}} \sum_{m=1}^{\infty}(m n)^{j}(e(m n \tau)+e(-m n \bar{\tau})) .(4.5)
\end{aligned}
$$

Differentiating both sides of (4.3) $j$ times gives

$$
\begin{aligned}
(2 \pi i)^{j} \sum_{m=1}^{\infty} m^{j} e(m \tau) & =\frac{i}{2}(\cot \pi \tau)^{(j)} \\
& =\frac{i}{2}(2 \pi)^{j}(-1)^{\left[\frac{j}{2}\right]} \sum_{l=0}^{\left[\frac{j+1}{2}\right]} C_{j+1-2 l}(\cot \pi \tau)^{j+1-2 l}
\end{aligned}
$$

that is

$$
\sum_{m=1}^{\infty} m^{j} e(m \tau)=\frac{(-1)^{j}}{2} i^{j+1}(-1)^{\left[\frac{j}{2}\right]} \sum_{l=0}^{\left[\frac{j+1}{2}\right]} C_{j+1-2 l}(\cot \pi \tau)^{j+1-2 l},
$$

where each coefficient $C_{j+1-2 l}$ can be expressed in terms of Stirling numbers of the second kind $[9$, p.37]. From (4.6), we have

$$
\begin{aligned}
& \sum_{m=1}^{\infty} m^{j}(e(m \tau)+e(m \bar{\tau})) \\
= & \frac{1}{2} i^{j+1}(-1)^{\left[\frac{j}{2}\right]} \sum_{l=0}^{\left[\frac{j+1}{2}\right]} C_{j+1-2 l}\left\{(-1)^{j}(\cot \pi \tau)^{j+1-2 l}-(\cot \pi \bar{\tau})^{j+1-2 l}\right\} .
\end{aligned}
$$


From (4.5) and (4.7), we have

$$
\begin{aligned}
\frac{1}{2} a^{k+1} Z(k+1)= & \zeta(2 k+2)+\frac{\pi i}{(2 y)^{2 k+1}}\left(\begin{array}{c}
2 k \\
k
\end{array}\right) \sum_{n=1}^{\infty} \frac{\cot n \pi \tau-\cot n \pi \bar{\tau}}{n^{2 k+1}} \\
& +\frac{\pi i}{(2 y)^{2 k+1}} \sum_{j=1}^{k}\left(\begin{array}{c}
2 k-j \\
k
\end{array}\right)(-1)^{\left[\frac{j}{2}\right]} \frac{1}{j !} i^{j}(4 \pi y)^{j} \sum_{l=0}^{\left[\frac{j+1}{2}\right]} C_{j+1-2 l} \\
& \cdot \sum_{n=1}^{\infty} \frac{(-1)^{j}(\cot \pi n \tau)^{j+1-2 l}-(\cot \pi n \bar{\tau})^{j+1-2 l}}{n^{2 k+1}}
\end{aligned}
$$

that is

$$
\begin{aligned}
\frac{1}{2} a^{k+1} Z(k+1)= & \zeta(2 k+2)+\frac{\pi i}{(2 y)^{2 k+1}} \sum_{j=0}^{k}\left(\begin{array}{c}
2 k-j \\
k
\end{array}\right)(-1)^{\left[\frac{j}{2}\right]}(4 \pi y)^{j} / j ! \\
& \cdot \sum_{l=0}^{\left[\frac{j+1}{2}\right]} C_{j+1-2 l} \sum_{n=1}^{\infty} \frac{(-1)^{j}(\cot \pi n \tau)^{j+1-2 l}-(\cot \pi n \bar{\tau})^{j+1-2 l}}{n^{2 k+1}} .
\end{aligned}
$$

In particular, if $b=0$, then $x=0, \tau=y i=\sqrt{\frac{c}{a}} i, \cot \tau=\cot (y i)=-i \operatorname{coth} y$, and

$$
\begin{aligned}
& \frac{1}{2} a^{k+1} Z(k+1) \\
& =\zeta(2 k+2)+\frac{2 \pi}{(2 y)^{2 k+1}} \sum_{j=0}^{k}(-1)^{\left[\frac{j+1}{2}\right]}\left(\begin{array}{c}
2 k-j \\
k
\end{array}\right)(4 \pi y)^{j} / j ! \\
& \quad \cdot \sum_{l=0}^{\left[\frac{j+1}{2}\right]} C_{j+1-2 l} \sum_{n=1}^{\infty} \frac{(\operatorname{coth} n \pi y)^{j+1-2 l}}{n^{2 k+1}} .
\end{aligned}
$$

\section{References}

[1] P. T. Bateman and E. Grosswald, "On Epstein's zeta function," Acta Arith., 9 (1964), 365-373.

[2] N. N. Lebedev, Special Functions and their Applications, Prentice-Hall Inc., Englewood Cliffs, N. J. (1965).

[3] Y. Motohashi, "A new proof of the limit formula of Kronecker," Proc. Japan. Acad., 44 (1968),614616.

[4] A. Selberg and S. Chowla, “On Epstein's zeta function," J. Reine Angew. Math., 227 (1967),86-110.

[5] C. L. Siegel, Advanced Analytic Number Theory, Tata Institute of Fundamental Research, Bombay (1980).

[6] J. R. Smart, "On the values of the Epstein zeta function," Glasgow Math. J., 14 (1973), 1-12.

[7] P. R. Taylor, "The functional equation for Epstein's zeta function," Quart. J. Math. Oxford Ser., 11 (1940), 177-182.

[8] E. C. Titchmarsh, The Theory of the Zeta-function, Clarendon Press, Oxford (1986).

[9] I. J. Schwatt, An Introduction to the Operations with Series, Cheesea Publishing Company, New York (1961). 
Information Department, The People's University of China, Beijing, People's Republic of China, 100872. Department of Mathematics and Statistics, Carleton University, Ottawa, Ontario, Canada, K1S 5B6. 